\title{
Análise do tempo até a re-hospitalização de pacientes com esquizofrenia via modelo de riscos proporcionais de Cox
}

\section{Analysis of the time to re-hospitalization of patients with schizophrenia via Cox proportional hazard model}

\author{
Marcia Araujo Maia'; Eduardo Yoshio Nakano²
}

\section{Resumo}

O objetivo desse trabalho foi obter um modelo que estime o tempo até a re-hospitalização de pacientes com esquizofrenia tratados por diferentes tipos de antipsicóticos. Essa informação é de grande importância, pois pode ser utilizada como uma medida de efetividade do medicamento e então auxiliar o psiquiatra na escolha do melhor tratamento a ser utilizado. A análise considerou uma amostra de 132 pacientes com esquizofrenia e foi realizada utilizando o modelo de riscos proporcionais de Cox. Toda a análise foi realizada pelo software livre R.

Palavras-chave: Análise de sobrevivência, dados censurados, antipsicóticos, medicamentos.

\begin{abstract}
The aim of this work was to obtain a model that estimates the time to re-hospitalization of patients with schizophrenia treated by several types of antipsychotics. This kind of information is very important because it can be used as a measure of effectiveness of the medication, therefore it can help the psychiatrist in choosing the best treatment to be used. The analysis examined a sample of 132 patients with schizophrenia and was performed using the Cox proportional hazard model. All analysis where performed by the free software R.
\end{abstract}

Keywords: Survival analysis, censored data, antipsychotics, medications.

1 Departamento de Estatística, Universidade de Brasília; maia.am@outlook.com

2 Departamento de Estatística da Universidade de Brasília; nakano@unb.br 


\section{Introdução}

O termo esquizofrenia vem do grego e significa mente dividida. Essa doença faz com que haja uma separação entre a imaginação e a realidade. Segundo Silva (2006), a definição atual indica uma psicose crônica idiopática, aparentando ser um conjunto de diferentes doenças com sintomas que se assemelham e se sobrepõem. Alguns dos sintomas da esquizofrenia são alucinações, delírios, alterações do comportamento, do pensamento, dentre outros.

Os pacientes com esquizofrenia precisam receber tratamento por toda vida, e algumas maneiras conhecidas para tratar a doença são o uso de medicação, a prática de terapia, e diversos outros métodos. No caso do tratamento por medicação, o tempo entre as rehospitalizações é um importante indicador de efetividade do medicamento. Assim, ter um modelo que estime corretamente o tempo até a re-hospitalização dos pacientes permitirá avaliar o grau de efetividade do medicamento administrado no paciente e decidir se é necessária, ou não, a sua substituição. Os diferentes tipos de antipsicóticos podem apresentar efeitos colaterais diversos e uma estimação precisa de sua eficiência permitirá a melhor escolha do medicamento para o paciente.

Neste contexto, o objetivo geral deste trabalho foi ajustar o modelo de riscos proporcionais de Cox que estime o tempo até a re-hospitalização de pacientes com esquizofrenia tratados por diferentes tipos de antipsicóticos.

A escolha do modelo de Cox neste trabalho foi devido ao fato desse modelo ser um dos mais populares na análise de dados de sobrevivência, principalmente na área de saúde. A principal razão dessa popularidade é a presença da componente não-paramétrica, que torna o modelo bastante flexível. Além disso, a proporcionalidade dos riscos é uma característica muito conveniente, principalmente quando o interesse é interpretar os coeficientes estimados.
Obter um modelo que estime com precisão o tempo entre as rehospitalizações dos pacientes, e identificar quais são os fatores que influenciam esse tempo, é de grande importância para medir a efetividade do tratamento, e pode auxiliar o médico psiquiatra na escolha do tratamento a ser utilizado. O estudo foi realizado dentro do contexto de Análise de Sobrevivência, utilizando o modelo de riscos proporcionais de Cox. Toda a análise foi realizada pelo software livre $R(R$ CORE TEAM, 2015).

\section{Metodologia}

\section{Descrição dos dados}

Os dados utilizados neste trabalho foram obtidos em um estudo realizado no Instituto de Psiquiatria (IPq) do Hospital das Clínicas (HC) da Universidade de São Paulo (USP), no período de 1 de dezembro de 1997 a 31 de dezembro de 2004. Este estudo teve como objetivo avaliar o tempo até a re-hospitalização de pacientes com esquizofrenia tratados por diferentes tipos de antipsicóticos. Resultados desse estudo, sem a consideração de covariáveis na análise, foram apresentados em Werneck et al (2011).

O presente trabalho teve a proposta de modelar o tempo até a re-hospitalização dos pacientes com esquizofrenia considerando a presença de oito covariáveis, descritas a seguir: 1 . Idade do paciente, em anos; 2. Sexo do paciente; 3. Idade de início da doença, em anos; 4. Duração da doença, em anos; 5. Número de internações do paciente; 6. Número de medicamentos utilizados; 7. Tempo de internação, em dias e; 8. Grupo de medicação utilizado.

As variáveis quantitativas consideradas no estudo foram dicotomizadas, agrupando seus níveis de acordo com seus riscos relativos. O procedimento consistiu em categorizar as variáveis de acordo com seus quantis e agrupar aqueles níveis que apresentaram riscos relativos individuais semelhantes. Além disso, a variável Grupo de 
Medicação considerou apenas dois grupos de antipsicóticos: Clozapina e Antipsicóticos de Segunda Geração (ASG).

A amostra foi composta por 132 pacientes, no qual $106(80,3 \%)$ foram observações censuradas. A distribuição de frequências das categorias das oito covariáveis é apresentada na Tabela 1 .

\section{Modelo Estatístico}

Os dados do tempo até a re-hospitalização dos pacientes foram analisados por meio de técnicas de Análise de Sobrevivência, em que $T$ é a variável que denota o tempo (em dias) compreendido entre o paciente ser medicamentado e voltar a ser internado. $\mathrm{O}$ tempo foi considerado censurado à direita em situações no qual, por algum motivo, foi perdido o acompanhamento do paciente durante o período de estudo ou o paciente não foi rehospitalizado até o dia 31 de dezembro de 2004.

Os dados foram modelados considerando o modelo semi-paramétrico de riscos proporcionais de Cox (COX, 1972). Este modelo propõe modelar os dados de sobrevivência, na presença de covariáveis, por meio da função de risco. Segundo o modelo de Cox (COX, 1972), a função de risco para um indivíduo com vetor de covariáveis $\mathbf{x}$ é dada por

$$
h_{\mathrm{Cox}}(t \mid \mathbf{x})=h_{0}(t) \exp \left\{\mathbf{x}^{\prime} \boldsymbol{\beta}\right\}
$$

em que $h_{0}(t)$ é conhecida como função de risco base, ou seja, o risco de um indivíduo com todas as covariáveis iguais a zero e $\beta$ é o vetor de coeficientes de regressão desconhecidos.

A função de sobrevivência para um indivíduo com vetor de covariáveis $\mathbf{x}$ está relacionada com a função de risco (1) e pode ser escrita como

$$
S_{\mathrm{Cox}}(t \mid \mathbf{x})=\left[S_{0}(t)\right]^{\exp \left\{\mathbf{x}^{\prime} \boldsymbol{\beta}\right\}}
$$

Aqui, $S_{0}(t)$ é a função de sobrevivência base, ou seja, a função de sobrevivência de um indivíduo com todas as covariáveis iguais a zero.

Tabela 1 - Frequências e percentuais das covariáveis consideradas

\begin{tabular}{|c|c|c|}
\hline Variável & $\mathbf{N}$ & $\%$ \\
\hline \multicolumn{3}{|l|}{ Idade (anos) } \\
\hline$<50$ & 118 & $89,4 \%$ \\
\hline$\geq 50$ & 14 & $10,6 \%$ \\
\hline \multicolumn{3}{|l|}{ Sexo } \\
\hline Masculino & 81 & $61,36 \%$ \\
\hline Feminino & 51 & $38,64 \%$ \\
\hline \multicolumn{3}{|c|}{ Idade de Início (anos) } \\
\hline$<20$ & 66 & $50,0 \%$ \\
\hline$\geq 20$ & 66 & $50,0 \%$ \\
\hline \multicolumn{3}{|l|}{ Duração (anos) } \\
\hline$<20$ & 94 & $71,21 \%$ \\
\hline$\geq 20$ & 38 & $28,79 \%$ \\
\hline \multicolumn{3}{|c|}{ Número de Internações } \\
\hline$<10$ & 124 & $93,94 \%$ \\
\hline$\geq 10$ & 8 & $6,06 \%$ \\
\hline \multicolumn{3}{|c|}{ Número de Medicamentos } \\
\hline$<3$ & 88 & $66,67 \%$ \\
\hline$\geq 3$ & 44 & $33,33 \%$ \\
\hline \multicolumn{3}{|c|}{ Tempo de Internação (dias) } \\
\hline$<80$ & 20 & $15,15 \%$ \\
\hline$\geq 80$ & 112 & $84,85 \%$ \\
\hline \multicolumn{3}{|l|}{ Grupo de Medicação } \\
\hline Clozapina & 42 & $31,82 \%$ \\
\hline $\mathrm{ASG}^{*}$ & 90 & $68,18 \%$ \\
\hline
\end{tabular}
no estudo.

Trabalhos que apresentam aplicações do modelo de riscos proporcionais de Cox podem ser vistos em Nakano e Cunha (2012), Santos e Nakano (2015), entre outros.

Inicialmente, o modelo de riscos proporcionais de Cox foi ajustado considerando todas as 8 covariáveis apresentadas pela Tabela 1 . O método de seleção Backward foi plicado para identificar as covariáveis significativas no modelo. Após a aplicação do método de seleção de variáveis, o modelo final contou apenas com aquelas covariáveis que apresentaram um nível de significância de 5\%. A suposição de proporcionalidade dos riscos foi verificada por meio dos resíduos escalonados de Schoenfeld (SCHOENFELD, 1982). O ajuste 
global do modelo final foi verificado pelo resíduo de Cox-Snell (COX e SNELL, 1968) e um possível problema de multicolinearidade das covariáveis do modelo foi verificado por meio do FIV - Fator Inflacionário da Variância (NETER et al., 1990).

\section{Resultados e discussões}

O modelo de riscos proporcionais de Cox foi ajustado para os dados do tempo atéa re-hospitalização e, após a seleção de variáveis descritas na Tabela 1, três das oito covariáveis inicialmente consideradas permaneceram no modelo. Os resultados são apresentados na Tabela 2.

Tabela 2 - Estimativas dos parâmetros do modelo de riscos proporcionais de Cox.

\begin{tabular}{|c|c|c|c|c|}
\hline Variável* & $\begin{array}{c}\beta \\
(\mathbf{E P})\end{array}$ & $\begin{array}{c}\mathbf{R R}^{* *} \\
\text { (IC 95\%) }\end{array}$ & $p$ & FIV \\
\hline \multicolumn{5}{|l|}{ Sexo } \\
\hline Masculino & 0 & 1 & & \\
\hline Feminino & $\begin{array}{c}0,832 \\
(0,395) \\
\end{array}$ & $\begin{array}{c}2,298 \\
(1,06-4,982) \\
\end{array}$ & 0,035 & 1,004 \\
\hline \multicolumn{5}{|c|}{ Número de Medicamentos } \\
\hline$<3$ & 0 & 1 & & \\
\hline$\geq 3$ & $\begin{array}{c}0,972 \\
(0,421)\end{array}$ & $\begin{array}{c}2,644 \\
(1,159-6,032)\end{array}$ & 0,021 & 1,370 \\
\hline \multicolumn{5}{|c|}{ Grupo de Medicação } \\
\hline Clozapina & 0 & 1 & & \\
\hline $\mathrm{ASG}^{* * *}$ & $\begin{array}{c}1,334 \\
(0,501) \\
\end{array}$ & $\begin{array}{c}3,799 \\
(1,423-10,14)\end{array}$ & 0,008 & 1,366 \\
\hline
\end{tabular}

"As categorias com coeficientes $\beta$ iguais a zero são os níveis de referência das variáveis; * ${ }^{*} R R=$ Risco Relativo; ${ }^{* * *}$ Antipsicóticos de segunda geração. Fonte: Os autores.

Observando os resultados da Tabela 2, vemos que as variáveis Sexo, Número de medicamentos e Grupo de medicação influenciaram de forma significativa o tempo até a re-hospitalização. Já as variáveis idade, idade de inicio da doença, duração da doença, número de internações e tempo de internação não apresentaram efeito significativo. Conforme os valores observados do FIV, não foram identificados problemas de multicolinearidade entre as covariáveis, podendo cada uma delas ser interpretada individualmente. Assim, segundo o modelo de Cox, o risco de rehospitalizaçção de um paciente do sexo feminino é 2,298 vezes (129,8\% maior) o risco de re-hospitalização de um paciente do sexo masculino. Aqueles pacientes que usam pelo menos 3 medicamentos apresentaram um risco de re-hospitalização 2,644 vezes $\quad(164,4 \%$ maior) o risco de pacientes que usam até dois medicamentos. O risco de re-hospitalização de pacientes que usam antipsicóticos de segunda geração é 3,799 (279,9\% maior) o risco daqueles pacientes que fazem uso de clozapina.

Segundo o modelo de Cox (COX, 1972), a função de sobrevivência (2), para um indivíduo com vetor de covariáveis $\mathbf{x}$, é estimada por:

$$
\hat{S}_{\mathrm{Cox}}(t \mid \mathbf{x})=\left[\hat{S}_{0}(t)\right]^{\exp \left\{\mathbf{x}^{\prime} \hat{\boldsymbol{\beta}}\right\}}
$$

Aqui, $\hat{S}_{0^{\prime}}$ é o vetor de parâmetros estimados do modelo de Cox, cujas estimativas são apresentadas pela Tabela 2 e $\hat{S}_{0}(t)$ é estimativa da função de sobrevivência base (função de sobrevivência de um indivíduo com todas as covariáveis iguais a zero), cujas estimativas são apresentadas pela Tabela 3.

Tabela 3 - Estimativas da função de sobrevivência base.

\begin{tabular}{cccccc}
\hline tempo & $\hat{S}_{0}(t)$ & tempo & $\hat{S}_{0}(t)$ & tempo & $\hat{S}_{0}(t)$ \\
\hline 1 & 1 & 106 & 0,9248 & 483 & 0,8475 \\
13 & 0,9939 & 110 & 0,9172 & 517 & 0,8360 \\
36 & 0,9877 & 113 & 0,9095 & 594 & 0,8241 \\
41 & 0,9687 & 226 & 0,9003 & 604 & 0,8120 \\
57 & 0,9619 & 350 & 0,8904 & 705 & 0,7995 \\
68 & 0,9548 & 362 & 0,8806 & 782 & 0,7863 \\
88 & 0,9474 & 412 & 0,8703 & 969 & 0,7728 \\
90 & 0,9325 & 421 & 0,8590 & 1033 & 0,7589 \\
\hline
\end{tabular}

Assim, de acordo com a função de sobrevivência estimada (3), temos que a probabilidade de uma 
mulher, que toma três ou mais medicamentos e que faz uso de Clozapina permanecer mais de 110 dias sem ser rehospitalizada é de:

$$
\hat{S}_{\mathrm{Cox}}(110 \mid \mathbf{x})=\left[\hat{S}_{0}(110)\right]^{\exp \{0,832+0,972+0\}}=0,8955
$$

A Figura 1, a seguir, apresenta a função de sobrevivência estimada para todas as oito $\left(2^{3}\right)$ possíveis combinações das covariáveis do modelo final.

Figura 1 - Estimativas das funções de sobrevivências segundo o modelo de riscos proporcionais de Cox.

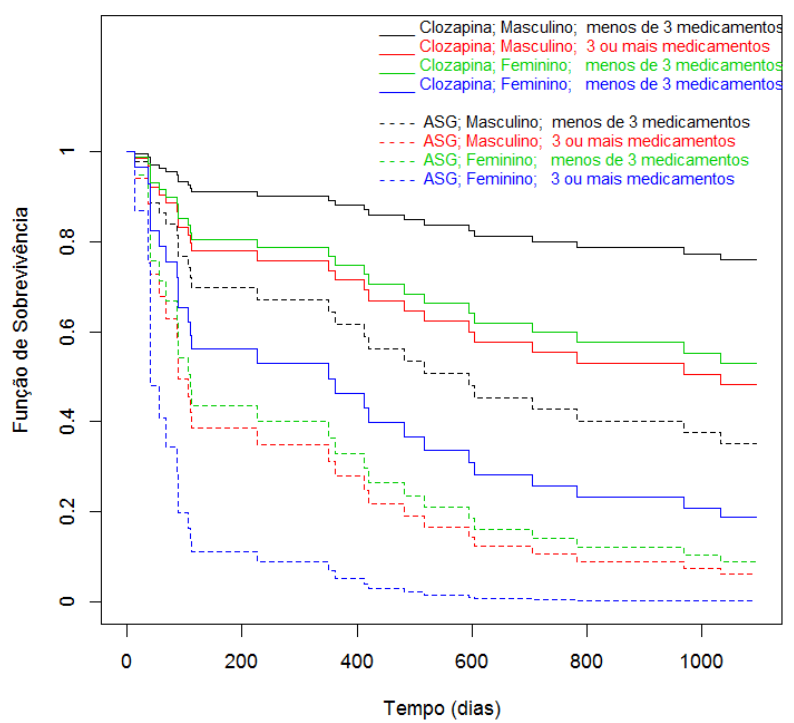

Fonte: Os autores.

A verificação da suposição básica do modelo de Cox, que assume proporcionalidade dos riscos, foi avaliada graficamente por meio dos resíduos escalonados de Schoenfeld e pelo teste da proporcionalidade da função de risco. O gráfico de resíduos de Schoenfeld é apresentado pela Figura 2 e a Tabela 4 apresenta os resultados do teste de tendências da função de risco.
Figura 2 - Resíduos escalonados de Schoenfeld. Sexo
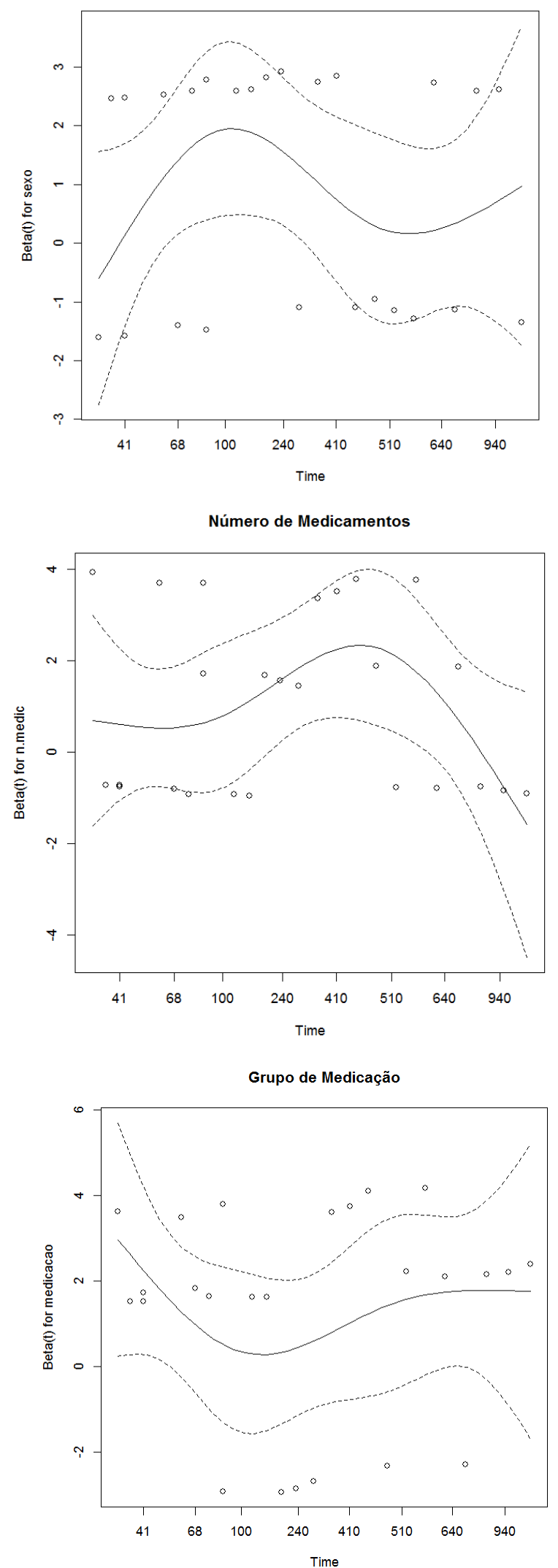

Fonte: Os autores. 
Tabela 4 - Testes de tendências.

\begin{tabular}{lccc}
\hline & $\boldsymbol{\rho}^{*}$ & $\chi^{2}$ & $\boldsymbol{p}$ \\
\hline Sexo & $-0,0150$ & 0,00575 & 0,940 \\
Número de Medicamentos & $-0,0210$ & 0,00923 & 0,923 \\
Grupo de Medicação & $-0,0233$ & 0,01204 & 0,913 \\
GLOBAL & NA & 2,4451 & 0,999 \\
\hline
\end{tabular}

Fonte: Os autores.

* coeficiente de correlação entre o tempo de sobrevivência e o resíduo de Schoenfeld.

Pode-se observar que não há tendências significativas, tanto para as três variáveis que permaneceram no modelo final quanto para $o$ modelo global. Nos gráficos apresentados pela Figura 2 é possível visualizar tais tendências não acentuadas ao longo do tempo, comprovando que a suposição de proporcionalidade dos riscos não está sendo violada na análise desses dados.

A verificação da qualidade do ajuste dos riscos proporcionais de Cox foi realizada por meio do resíduo de Cox-Snell (COX e SNELL, 1968). Os resultados são apresentados pela Figura 3.

Segundo Lawless (2003), os resíduos de CoxSnell vêm de uma população homogênea e devem seguir uma distribuição exponencial com média 1. Quanto mais a função de sobrevivência dos resíduos do modelo de Cox se aproxima da função de sobrevivência da exponencial, melhor é o ajuste do modelo de Cox. Segundo Klein e Moeschberguer (2003), essa interpretação deve ser feita com cautela, visto que a distribuição exponencial dos resíduos somente é válida quando os verdadeiros valores dos parâmetros são considerados. Desvios da distribuição exponencial podem ocorrer, em algumas situações, devido à incerteza no processo de estimação dos parâmetros. E, esses desvios podem ser maiores, principalmente, na cauda direita da distribuição e em casos de amostras pequenas.
Figura 3 - Comparações das funções de sobrevivência do resíduo de Cox-Snell com a Exponencial.
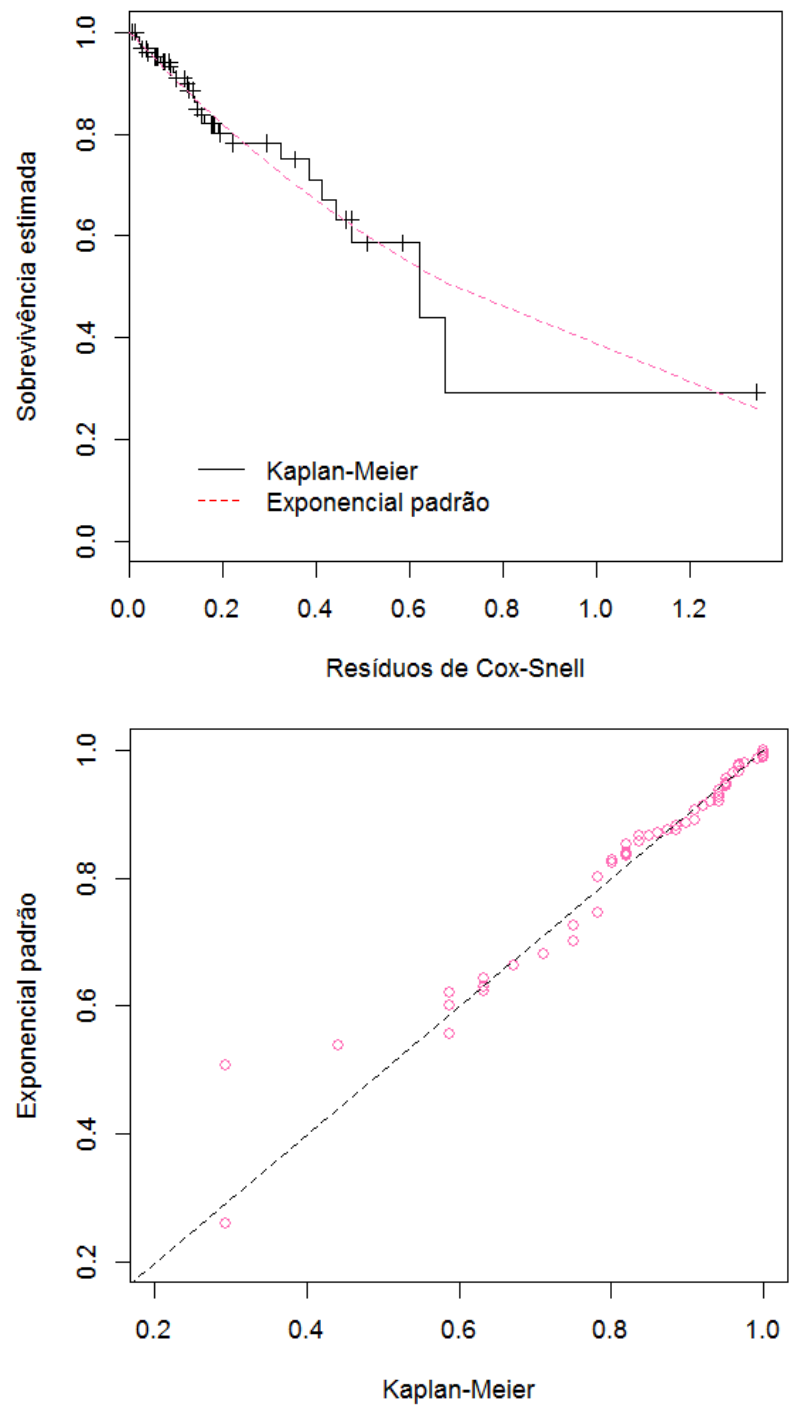

Fonte: Os autores.

Pode ser observado, a partir da Figura 3, que a função de sobrevivência dos resíduos se ajustou bem à função de sobrevivência da exponencial padrão, sobretudo para os valores pequenos dos resíduos. Esse resultado é um indicativo do bom ajuste do modelo de Cox, que pode ser confirmado pelo teste de Kolmogorov-Smirnov (CONOVER, 1999). O teste de Kolmogorov-Smirnov, para verificação do ajuste da distribuição Exponencial padrão dos resíduos, resultou na estatística do teste $D=0,215$. Considerando um nível de significância de $5 \%$ e $n=26$ (26 valores não censurados), o valor crítico do 
teste de Kolmogorov-Smirnov é igual a $D_{\text {crítico }}=0,259$, não rejeitando a hipótese do resíduo de Cox-Snell seguir uma distribuição exponencial padrão.

Note que o alto valor $(D=0,215)$ da diferença entre as funções de sobrevivência observada na Figura 3, percebido na cauda direita da distribuição dos resíduos, é um fenômeno já previsto por Moeschberguer. Visto que as observações (neste caso os resíduos de Cox-Snell) vão experimentando o evento de interesse ou sendo censurados ao longo do tempo, o número de observações na cauda da função de sobrevivência é pequeno. Neste contexto, os desvios presentes na cauda da função de sobrevivência não devem ser tratados da mesma forma que os desvios das funções nos valores iniciais, corroborando ainda mais a suposição de bom ajuste do modelo de riscos proporcionais de Cox para os dados dessa aplicação.

\section{Conclusões}

O modelo de riscos proporcionais de Cox mostrou-se adequado para ajustar a distribuição do tempo até a re-hospitalização de pacientes com esquizofrenia. Dentre as 8 variáveis, inicialmente consideradas na análise, três delas (sexo, número de medicamentos e grupo de medicação) foram significativas. Além disso, foi verificado que não há multicolinearidade entre as variáveis avaliadas.

Os resultados mostraram que as mulheres apresentaram um menor tempo (maior risco) até a re-hospitalização. Com relação ao número de medicamentos, pacientes que faziam uso de três ou mais medicamentos apresentam menor tempo até a re-hospitalização, quando comparados com aqueles que faziam uso de menos de três medicamentos. Ainda, pacientes que fizeram uso de antipsicóticos de segunda geração também apresentaram menores tempos até a re-hospitalização do que aqueles pacientes que fizeram uso de Clozapina.

Como propostas futuras de trabalho, modelos de regressão paramétricos podem ser considerados como alternativas ao modelo de riscos proporcionais de Cox.
Apesar do tempo considerado ser medido em dias, Nakano e Carrasco (2006) mostraram que quando a variabilidade dos dados é grande, um modelo contínuo pode ser utilizado para modelar dados discretos.

\section{Referências}

CONOVER, W. J. Practical nonparametric statistics. 3. ed. New York: J Wiley, 1999.

COX, D. R. Regression models and life tables (with discussions). J.R. Stat. Soc. B, v.34, n.2, p.187-202, 1972.

COX, D. R.; SNELL, E. J. A general definition of residuals. J.R. Stat. Soc. B, v.30, n.2, p.248-275, 1968.

KAPLAN, E. L.; MEIER, P. Nonparametric estimation from incomplete observations. J. Am. Stat. Assoc., v.53, n.282, p.457-481, 1958.

KLEIN, J. P; MOESCHBERGER, M. L. Survival analysis: Techniques for censored and truncated data. 2.ed. New York: Springer-Verlag, 2003.

LAWLESS, J. F. Statistical models and methods for lifetime data. 2.ed. New Jersey: John Wiley \& Sons, 2003.

NAKANO, E. Y.; CARRASCO, C. G. Uma avaliação do uso de um modelo contínuo na análise de dados discretos de sobrevivência. TEMA - Tend. Mat. Apl. Comput., v.7, n.1, p.91-100, 2006.

NAKANO, E. Y.; CUNHA, J. F. Análise do efeito da camuflagem no tempo de segregação em regiões texturizadas utilizando o modelo de riscos proporcionais de Cox. Semina: Ciências Exatas e Tecnológicas, v.33, n.2, p.141-148, 2012.

NETER, J.; WASSERMAN, W.; KUTNER, M. H. Applied Linear Regression Models. 3.ed, Homewood, IL: Irwin, 1990.

R CORE TEAM (2015). R: A language and environment for statistical computing. R Foundation for Statistical Computing, Vienna, Austria. ISBN 3-900051-07-0, URL http://www.R-project.org. 
SANTOS, R. O; NAKANO, E. Y. Análise do tempo de permanência de trabalhadores no mercado de trabalho do Distrito Federal via modelo de riscos proporcionais de Cox e Log-normal. Rev. Bras. Biom., v.33, n.4, p.570-584, 2015.

SCHOENFELD, D. Partial Residuals for the proportional hazard regression model. Biometrika, v.69, n.1, p.329-241, 1982.

SILVA, R. C. B. Esquizofrenia: uma revisão. Psicol. USP, v.17, n.4, p.263-285, 2006.

WENECK, A.; HALLAK, J.; NAKANO, E. Y; ELKIS, H. Time to rehospitalization in patients with schizophrenia discharged on first generation antipsychotics, non-clozapine second generation antipsychotics, or clozapine. Psychiatry Research, v.188, n.3, p.315-319, 2011. 